\title{
Assessment of Knowledge, Attitude and Practice with Regard to Periodontal Health Assessment and Plaque Control Measures among Dental Graduates in Mumbai, India-A Descriptive Cross-Sectional Study
}

\author{
Gulnar Dara Sethna ${ }^{*}$, Priyanka Sambhaji Machale², Surabhi Sanat Nabazza ${ }^{3}$ and Rajesh Prabhakar \\ Gaikwad $^{4}$
}

${ }^{1}$ Assistant Professor, Department of Periodontology, Government Dental College and Hospital, Mumbai, India

${ }^{2}$ Assistant Professor, Department of Public health dentistry, Government Dental College and Hospital, Mumbai, India

${ }^{3}$ Private practice, Mumbai, India

${ }^{4}$ Associate Professor and Head of the Department of Periodontology, Government Dental College and Hospital, Mumbai, India

*Corresponding author: Gulnar Dara Sethna, Assistant Professor, Department of Periodontology, Government Dental College and Hospital, Mumbai, India.
Received Date: July 28, 2020

Published Date: August 20, 2020

\section{Abstract}

Background: Effective mechanical and chemical plaque control remain the cornerstone of successful periodontal treatment and can be accomplished with the general awareness of the oral hygiene aids.

Aim \&Objectives: To assess the knowledge, attitude and practice among graduate dentists in dental clinics with regard to periodontal health assessment and plaque control practices in patients.

Materials and Methods: This cross-sectional multi-centre, close-ended questionnaire -based survey collected data by convenience sampling from 400 graduate dentists with minimum of 5 years of dental practice in Mumbai, India. 392 responses met the inclusion criteria and were included in the study. A well-structured 20-item questionnaire was prepared and validated. The questionnaire included questions pertaining to assessment of knowledge, attitude and oral hygiene practices among dentists with respect to periodontal health assessment and plaque control in dental clinics in Mumbai.

Results: Our questionnaire-based survey revealed that there was general consensus among the dentists about plaque control measures like tooth-brushing/interdental cleansing aids and recall visits for patients. However there were diversity and ambiguity in responses among dentists about periodontal assessment by perio charting, the use of mouthwashes, brushing techniques, gum paints and periodontal maintenance in implant patients.

Conclusion: There was positive attitude and awareness among dentists in this survey about the assessment of periodontal health, plaque control measures and patient education in dental practice. However, instilling the knowledge of the correct oral hygiene practices is the need of the hour and needs to be implemented at the undergraduate level. The results of this present study re-enforces the belief that the knowledge and attitude of the general dentists towards oral hygiene practices are of paramount importance and ultimately translate into optimum patient care. 


\section{Introduction}

India is the second most populous country after China equivalent to $17.7 \%$ of the total world population and is soon projected to become the most populous country in the world with nearly 1.5 billion population. The prevalence of periodontal diseases ranges from 50 to $100 \%$ in various parts of the Indian subcontinent [1]. In developing countries due to the high prevalence, they have serious socio-economic repercussions and adversely affect the quality of life [2]. Dental plaque or biofilm and plays a pivotal role in the initiation and progression of periodontal and peri-implant diseases as shown by several epidemiological surveys. Hence disruption of the biofilm to prevent periodontal disease remains the gold standard for their primary treatment [3].

Periodontal diseases can be prevented by effective mechanical and chemical plaque control and begins with the correct periodontal assessment, proper tooth brushing and flossing techniques, periodic dental recall visits and appropriate dietary practices [4]. The dentists play an integral role in patient education and creating awareness about preventive measures for periodontal disease and for achieving good oral health. However, limited literature is available regarding the same. Therefore, this survey was conducted to assess the knowledge, attitude and practice of graduate dentists practicing in private clinics in Mumbai.

\section{Materials and Methods}

The study was designed as a descriptive cross-sectional survey which was conducted between December 2017 to August 2018. A well-structured, close- ended questionnaire comprising of 20 questions was prepared through multiple phases including experts review of the questions. A pilot study was conducted among 50 graduate dental practitioners in Mumbai by simple random sampling through the lottery method. The questionnaire was validated by testing it for its construct, content and face validity by the experts. Test re-test reliability was checked on ten samples and the reliability was found to be good (0.8)

The sample size was calculated on the basis of the formula as mentioned below:

$$
\begin{aligned}
& \text { Sample size: } \\
& \mathrm{n}=\mathrm{Z} 2 \mathrm{p}(1-\mathrm{p}) / \mathrm{d} 2 \\
& \mathrm{z}=\text { standardized normal deviate ( } \mathrm{z} \text { value) } 1.96 \text { for } 95 \% \\
& \text { confidence interval }
\end{aligned}
$$$$
\mathrm{p}=\text { prevalence of interest ( } 50 \% \text { as per pilot study) }
$$$$
\mathrm{d}=\text { clinically expected variation } 10 \% \text { of prevalence of interest }
$$$$
\text { Sample size }=(1.96) 2 \times 0.50 \times 0.50 / 0.52=384
$$

At 95\% confidence level and 5\% allowable error, the sample size was calculated to 384 and was rounded off to 400 participants.
Prior to conducting the the study, Ethical clearance was obtained from the Ethics review and research board of Government Dental College and Hospital, Mumbai and included confidentiality of the respondents and their informed consent. The inclusion criteria of study sample were dental surgeons with BDS Degree registered under the Maharashtra state dental council and having a minimum of five years experience of dental practice in dental clinics. Postgraduate dental surgeons were excluded to reduce the bias in the study. The list of all the registered private dental practitioners was obtained from the state directories of the Indian Dental Association and participants from private dental clinics were selected from all six zones of Mumbai city based on simple random sampling through the lottery method.

The questionnaire was e-mailed individually to all the 400 participating dental surgeons so as to maintain confidentiality and eliminate bias in the study. However, 392 participants met the inclusion criteria and were included in the study. The questions were related to periodontal health assessment and oral hygiene awareness among graduate dentists in dental clinics. Questions under a similar domain in relation to patients undergoing orthodontic treatment and tooth/implant borne prosthesis were also included in the questionnaire.

\section{Data Analysis}

The entire collected questionnaires were compiled, and data was analysed by using a SPSS software version 18 by using descriptive statistics.

\section{Results}

A total of 392 practitioners selected for the study, 208 female and 184 males, with a mean age of $38 \pm 14$, participated in the study with a response rate of $95.88 \% .86 .2 \%$ dentists included periodontal assessment during their routine consultation with $66.8 \%$ of them routinely recommending the use of interdental cleansing aids to patients. Regarding prescription of mouthwashes, $61.9 \%$ of dentists advised them to some periodontally compromised patients, whereas only $32.2 \%$ of dentists recommended them to all periodontally compromised patients. There was ambiguity seen with respect to the use of chlorhexidine mouthwashes; $24 \%$ used a chlorhexidine (CHX) mouthwash diluted with water while $60 \%$ of the dentists did not give a pre-procedural rinse to their patients.

$82.2 \%$ of the dentists recommended the ideal two minutes duration for brushing to their patients; $9.2 \%$ recommended brushing for more than two minutes and $8.6 \%$ of the dentists in the survey recommended brushing for one minute. In this survey, patients undergoing fixed orthodontic treatment were advised mandatory use of fluoridated toothpastes and mouthwashes by only $41.9 \%$ of the dentists. There was a variation seen amongst the dentists advising brushing techniques to their patients; $55 \%$ advised the Charter's method, $16.5 \%$ advised the Modified Bass 
technique while $28.5 \%$ of the dentists did not advise any particular brushing technique to their patients. There was also no unanimous practice with regard to the recommendation of frequency of tooth brushing for such patients. $49.7 \%$ of dentists advised tooth brushing twice a day while $47 \%$ advised their patients to brush their teeth after every meal.

Majority of the dentists recommended the use of powered toothbrushes with interdental cleansing aids to patients with fixed prosthesis and/or undergoing fixed orthodontic therapy (88\%). $41 \%$ of the dentists in the survey always prescribed fluoride toothpastes/mouthwashes to their patients who were undergoing orthodontic treatment. $73.8 \%$ of the dentists in the survey used special instruments like Implacare for supportive periodontal therapy in implant patients, whereas $26.2 \%$ still used ultrasonic scalers with stainless steel inserts. As recorded, $33.8 \%$ recommended Waterpik/Hydrofloss to all the patients, whereas $42.2 \%$ recommended waterpik/Hydrofloss only in periodontally compromised patients. There was a substantial majority of dentists $(70.5 \%)$ who advocated the use of gum paints for their periodontally compromised patients before treatment. In this survey, most dentists $(65 \%)$ recalled their patients within 3 months, with few dentists (20\%) even recalling patients within 1-2 months of periodontal therapy.

Radiographic examination during recall should be individualized so as to compare the initial findings with the post treatment findings. $86.8 \%$ of dentists agreed to a mandatory radiographic examination (Panoramic/IOPA) of the concerned area during recall. Perio-charting refers to a diagnostic chart used by dentists to record the gingival and periodontal status of the patients. It was routinely used for all patients by only $20 \%$ of the dentists. According to the survey based on the questionnaire, majority of the dentists routinely recommended the use of anticalculus toothpastes for plaque control (61.6\%). However,25\% of the dentists were not aware of them. There was also variation in consensus with $44.9 \%$ dentists stating that scaling and root planing was best done by a periodontist, $32.4 \%$ of them ascribing it to any dentist and $22.7 \%$ to a dental hygienist. Disclosing agents were used by only $18 \%$ of the dentists for motivating the patients as an adjunct to plaque control; however $60 \%$ of the dentists were unaware about them.

\section{Discussion}

Periodontal disease in our country has a higher prevalence rate with a negative impact on the life of individuals affected by it [4]. It is a well-documented that periodontal disease is the major cause of tooth loss in adults [5]. In addition, epidemiological surveys have shown that the dental plaque or biofilm plays an important role in the etiology of periodontal disease, hence removal of plaque biofilm is imperative to maintain a healthy periodontium $[1,6]$. Various systematic reviews support the role of mechanical and chemical plaque control in the reduction of plaque levels $[2,7]$. Dentists as health professionals of the future, play a primary role in maintenance of periodontal health by recommending the use of correct brushing techniques, routine use of interdental brushing aids, use of fluoridated dentifrices, and the judicious use of antibacterial mouthwashes. It is imperative that dentists should motivate and guide the patients towards effective plaque control. Dental and periodontal diseases can be preventable if the general dentists are knowledgable and have the right attitude to dental treatment [7].

A comprehensive periodontal assessment of the oral cavity plays a pivotal role in the diagnosis of the underlying disease condition and formulating the correct treatment plan for the patients. This includes diagnostic X-rays, study models, thorough examination of periodontal pockets, tooth mobility, recession etc. In our survey, $86.2 \%$ of the dentists included periodontal assessment in their routine consultations. Tooth brushes when used alone may be inaccessible for cleaning of interdental areas. To overcome these limitations, interdental cleansing aids like dental floss, toothpicks, Waterpik and interproximal brushes are highly recommended. According to some studies, there is a marked improvement in the periodontal health when interdental cleansing aids are used as adjuncts to conventional tooth brushing [8]. However, some studies conducted in India showed that majority of the patients were unaware of the interdental cleansing aids $[9,10]$. Interestingly, our survey showed that only $66.8 \%$ of the dentists recommended the use of interdental cleansing aids to their patients.

Oral irrigation effectively disrupts the subgingival plaque, cleaning non- adherent bacteria and debris from inaccessible areas and for delivers anti-microbial agents into periodontal pockets [11]. They are extremely important for the effective removal of food debris from posterior areas and in cases of fixed bridges or orthodontic appliances where the interdental cleaning devices may remain inaccessible [12]. According to a report of the American Academy of Periodontology (2001), oral irrigators are capable of decreasing gingival inflammation beyond that normally achieved by tooth brushing alone [13]. In our survey, 33.8\% of the dentists recommended the Waterpik/Hydro floss to all their dental patients, $42.2 \%$ recommended only them to periodontally compromised patients; however, some dentists (7.2\%) were unaware of oral irrigators.

The fixed orthodontic appliances such as brackets, arches wires, elastics etc. are plaque retentive areas in the mouth [14]. Hence, stringent plaque control measures like the triple- headed orthodontic toothbrushes, interdental cleansing aids coupled with proper frequency and technique of brushing and adjunctive use of fluorides (fluoride dentifrices/mouthwashes) play an important role in maintaining good oral hygiene in orthodontic patients [5]. In this survey, patients undergoing fixed orthodontic treatment were advised mandatory use of fluoridated toothpastes and mouthwashes by only $41.9 \%$ of the dentists. 
It is generally recommended to brush twice a day to disrupt the bacteria from repopulating within the stipulated $12 \mathrm{~h}$ cycle [15]. In our survey, it was found that there was no unanimous practice in regards with the recommendation of frequency of brushing for patients; $52.7 \%$ of dentists advised brushing teeth twice a day while $45.3 \%$ advised brushing of teeth after every meal. There were varied responses seen amongst the dentists regarding the brushing techniques. $55 \%$ of the dentists in the survey advised the Charter's method, $16.5 \%$ advised Modified Bass technique while a section of the dentists $(28.5 \%)$ did not recommend any particular brushing technique. These results are in concensus with the study conducted by Gupta et al who had observed that dentists too were unaware of the oral hygiene practices [16].

Chlorhexidine (CHX) molecule is a is a broad-spectrum antimicrobial agent and is considered as the gold standard as an anti-plaque agent [15]. Different concentrations of chlorhexidine $(0.2 \%$ and $0.02 \%)$ have been used as pre-procedural rinses to reduce the bioaerosol contamination in dental practice. Clinical studies have confirmed that $0.12 \%$ CHX gluconate has an equal therapeutic benefit as $0.2 \% \mathrm{CHX}$ at reducing the salivary bacterial load by $97 \%$. However, a lower concentration of CHX is associated with fewer side effects like mucosal erosion, tooth staining and taste alteration while maintaining the comparable therapeutic effect [17]. There was ambiguity seen with respect to the use of chlorhexidine mouthwashes; $24 \%$ used CHX mouthwashes diluted with water, alarmingly $60 \%$ of the dentists did not give a preprocedural rinse to their dental patients.

Long-term stability after periodontal treatment is as critically important in dental implants as it is for natural teeth $[18,19]$. This can be achieved with a disciplined maintenance care regimen and meticulous oral hygiene. Based on Merin's criteria for recall of patients after periodontal therapy, studies have convincingly demonstrated that a 3-month recall interval provides stability while intervals greater than six months would increase risk of periodontal disease recurrence [20-22]. In the present survey, 65\% of dentists scheduled a recall for their patients within 3 months after periodontal therapy, 15\% recalled within 6 months and $20 \%$ scheduled a recall within 1-2 months.

\section{Table}

\begin{tabular}{|c|c|c|c|}
\hline S. No & Knowledge attitude and Practice & Options & Responses \\
\hline \multirow{2}{*}{1} & \multirow{2}{*}{$\begin{array}{l}\text { Does routine consultation in your practice involve primary assessment } \\
\text { of periodontal health? }\end{array}$} & a) Yes & $86.20 \%$ \\
\hline & & b) No & $13.80 \%$ \\
\hline \multirow{3}{*}{2} & \multirow{3}{*}{ Do you advise use of interdental cleansing aids for your patients? } & a) Never & a) 0 \\
\hline & & b) Always & b) $66.8 \%$ \\
\hline & & c) Sometimes & c) $33.2 \%$ \\
\hline \multirow{4}{*}{3} & \multirow{4}{*}{ Do you routinely advise use of mouth washes to your patients? } & a) All dental patients & a) $4 \%$ \\
\hline & & b) All periodontally compromised patients & b) $32.2 \%$ \\
\hline & & c) Some periodontally compromised patients & c) $61.9 \%$ \\
\hline & & d) Do not prescribe mouthwash & d) $1.9 \%$ \\
\hline
\end{tabular}

Scaling around dental implants using the conventional metallic instruments leads to scratches, roughened surfaces, galvanic reactions between implant abutments and contamination on the implant surfaces thus making them more susceptible to adherence of plaque [23]. The titanium surface of implants entails scaling around the implants with special instruments like Colorvue probes, gold-plated curettes, unfilled plastic resin instruments (Implacare) and non-abrasive polishing pastes [24,25]. Use of power-driven instruments include the use of graphite piezo scaling tips and magneto-resin implant sheathed power scalers. Our survey recorded that $73.8 \%$ of dentists practiced a special implant care routine. However, $26.2 \%$ of the dentists still use ultrasonic scalers with stainless steel inserts which might roughen the implant surfaces contributing to plaque retention and peri-implantitis.

Perio charting is a very invaluable tool for a more comprehensive assessment of a patient's periodontal status and may be used to record pocket depth, clinical attachment loss, bleeding on probing, mobility, furcation, etc. However, in our survey, only $20 \%$ of the dentists routinely did a perio-charting for the periodontally compromised patients. The limitations of the present survey were that since it was a cross -sectional study which included the dentists only at Mumbai, it is not a true representation of all the dentists in the country.

\section{Conclusion}

The results of this present study re-enforces the belief that the knowledge and aptitude of the general dentists towards oral hygiene practices is of paramount importance. In our survey, several dentists were unaware about the basic plaque control protocols including the brushing techniques, interdental cleansing aids, perio-charting, protocols in periodontal maintenance in natural teeth/dental implants. It is the need of the hour to conduct awareness programs, seminars, dental camps etc. so as to update the knowledge of the dentists about concepts and innovations in oral hygiene practices based on evidence -based dentistry. This would in turn translate into better clinical practice and improved oral health of the society. It is highly recommended for a need for improvement and promulgation of optimal oral hygiene practices at the undergraduate curriculum and continuing education (Table 1). 


\begin{tabular}{|c|c|c|c|}
\hline \multirow{3}{*}{4} & \multirow{3}{*}{$\begin{array}{l}\text { How would you use chlorhexidine mouthwash as a pre-procedural rinse } \\
\text { for the patient? }\end{array}$} & a) $0.12 \% / 0.2 \%$ after dilution with water & $24 \%$ \\
\hline & & b) $0.12 \% / 0.2 \%$ without dilution with water & $16 \%$ \\
\hline & & c) Do not advise a pre-procedural rinse & c) $\quad 60 \%$ \\
\hline \multirow{2}{*}{5} & \multirow{2}{*}{$\begin{array}{l}\text { Do you demonstrate use of a different brushing technique to all patients } \\
\text { according to their periodontal status? }\end{array}$} & a) Yes & a) $68 \%$ \\
\hline & & b) No & b) $32 \%$ \\
\hline \multirow{3}{*}{6} & \multirow{3}{*}{$\begin{array}{l}\text { Which plaque control aids do you advice in patients undergoing fixed } \\
\text { orthodontic treatment/fixed denture prosthesis? }\end{array}$} & a) Only manual Tooth brushing & a) $10 \%$ \\
\hline & & b) Powered toothbrushes & b) $2 \%$ \\
\hline & & $\begin{array}{l}\text { c) Powered toothbrushes + interdental cleansing } \\
\text { aids }\end{array}$ & c) $88 \%$ \\
\hline \multirow{3}{*}{7} & \multirow{3}{*}{$\begin{array}{l}\text { Do you advice use of fluoridated toothpastes /mouthwashes in patients } \\
\text { undergoing fixed orthodontic treatment? }\end{array}$} & a) Always & a) $41 \%$ \\
\hline & & b) Sometimes & b) $55 \%$ \\
\hline & & c) Never & c) $4 \%$ \\
\hline \multirow{3}{*}{8} & \multirow{3}{*}{ Which tooth brushing technique do you advice your patients. } & b) Modified Bass method & a) $\quad 16.5 \%$ \\
\hline & & c) Charter's method & b) $55 \%$ \\
\hline & & d) No particular technique & c) $28.5 \%$ \\
\hline \multirow{3}{*}{9} & \multirow{3}{*}{$\begin{array}{l}\text { What frequency of toothbrushing do you recommend in patients under- } \\
\text { going fixed orthodontic treatment? }\end{array}$} & a) Once a day & a) $2 \%$ \\
\hline & & b) Twice a day & b) $52.7 \%$ \\
\hline & & c) After every meal & c) $45.3 \%$ \\
\hline \multirow{2}{*}{10} & \multirow{2}{*}{$\begin{array}{l}\text { Plaque control measures for patients receiving fixed partial denture in } \\
\text { your practice includes use of }\end{array}$} & a) Toothbrush only & a) $12 \%$ \\
\hline & & b) Toothbrush with interdental cleansing aids & b) $88 \%$ \\
\hline & & a) Ultrasonic scalers with stainless steel insert. & a) $26.2 \%$ \\
\hline 11 & What type of Scaling procedure for implant patients used in clinic & $\begin{array}{l}\text { b) Use of special scaling instruments for scaling in } \\
\text { implants (Implacare, plastic instruments etc.) }\end{array}$ & b) $73.8 \%$ \\
\hline & & a) One minute & a) $8.6 \%$ \\
\hline 12 & What is your recommended time for tooth brushing? & b) 2 minutes & b) $82.2 \%$ \\
\hline & & c) $>2$ minutes & c) $\quad 9.2 \%$ \\
\hline & & a) All patients & a) $33.8 \%$ \\
\hline & & b) All periodontally compromised patients & b) $42.2 \%$ \\
\hline 13 & Which patients would you recommend Waterpik/ Hydro floss to? & c) Patients with dental implants & c) $16.8 \%$ \\
\hline & & d) Not aware of Waterpik/Hydrofloss & d) $7.2 \%$ \\
\hline & & a) For all dental patients & a) $\quad 9 \%$ \\
\hline 14 & When do you advocate the use of Gum paints for the patients? & $\begin{array}{l}\text { b) For all periodontally compromised patients } \\
\text { before treatment. }\end{array}$ & b) $\quad 70.5 \%$ \\
\hline & & $\begin{array}{l}\text { c)For all periodontally compromised patients } \\
\text { after treatment }\end{array}$ & c) $\quad 20.5 \%$ \\
\hline 15 & Is an IOPA X-ray included as part of the periodontal recall visits? & $\begin{array}{l}\text { a) All patients undergo a mandatory radiographic } \\
\text { examination of the concerned area during recall. }\end{array}$ & a) $86.8 \%$ \\
\hline & & b) No radiographic examination required. & b) $13.2 \%$ \\
\hline & & a) $1-2$ months & a) $20 \%$ \\
\hline 16 & $\begin{array}{l}\text { When do you schedule a recall visit for your patients after periodontal } \\
\text { therapy? }\end{array}$ & b) Three months & b) $65 \%$ \\
\hline & & c) Six months & c) $15 \%$ \\
\hline & & a) Always & a) $20 \%$ \\
\hline 17 & $\begin{array}{c}\text { Do you use Perio charting for recording periodontal status of all pa- } \\
\text { tients? }\end{array}$ & b) Never & b) $31.4 \%$ \\
\hline & & c) Sometimes & c) $48.6 \%$ \\
\hline & & a) Always & a) $18 \%$ \\
\hline 18 & $\begin{array}{l}\text { Do you use disclosing agents for motivating your patient for plaque } \\
\text { control? }\end{array}$ & b) Never & b) $60 \%$ \\
\hline & & c)Sometimes & c) $22 \%$ \\
\hline & & a) Always & a) $61.6 \%$ \\
\hline 19 & $\begin{array}{l}\text { Do you routinely recommend the use of anti-calculus containing tooth- } \\
\text { pastes for plaque control? }\end{array}$ & b) Sometimes & b) $13.4 \%$ \\
\hline & & c)Not aware of them & c) $25 \%$ \\
\hline
\end{tabular}


Who in your opinion should do the scaling and root planning for patients in clinical practice?

\begin{tabular}{|c|c|}
\hline a) Any trained dentist & a) $32.4 \%$ \\
\hline b) A Dental hygienist & b) $22.7 \%$ \\
\hline c) Only by a Periodontist & c) $44.9 \%$ \\
\hline
\end{tabular}

\section{Acknowledgement}

None.

\section{Conflict of Interest}

No conflict of interest.

\section{References}

1. Dahlén G, Konradsson K, Eriksson S, Teanpaisan R, Piwat S, et al. (2010) A microbiological study in relation to the presence of caries and calculus. Acta Odontol Scand 68(4): 199-206.

2. Needleman I, Garcia R, Gkranias N, Kirkwood KL, Kocher T, et al. (2018) Mean annual attachment, bone level, and tooth loss: A systematic review. J Clin Periodontol 89: S120-S139.

3. Agarwal V, Khatri M, Singh G, Gupta G, Marya CM (2010) Prevalence of periodontal diseases in India. J Oral Health Community Dent 4: 7-16.

4. Azodo CC, Unamatokpa B (2012) Gender difference in oral health perception and practices among Medical House Officers. Russian Open Med J 1: 1-4.

5. Baelum V, López R (2013) Periodontal disease epidemiology-learned and unlearned? Periodontol 2000 62(1): 37-58.

6. Chapple IL, Van der Weijden F, Doerfer C, Herrera D, Shapira L, et al. (2015) Primary prevention of periodontitis: managing gingivitis. J Clin Periodontol 42 (16): S71-S76.

7. Gholami M, Pakdaman A, Jafari A, Virtanen JI (2014) Knowledge of and attitudes towards periodontal health among adults in Tehran. East Mediterr Health J 20(3): 196-202.

8. Charu, Madan (2014) A knowledge, attitude, and practices study regarding dental floss among dentists in India. J Indian Soc Periodontol 18(3): 361-368.

9. SN Goryawala, Paragkumar Chavda, Sneha Udhani, Naiya V Pathak, Shivang Pathak, et al. (2016) A survey on oral hygiene methods practiced by patients attending Dentistry Department at a Tertiary Care Hospital from Central Gujarat. J Int Soc Prev Community Dent 6(2): 115-119.

10. Oberoi SS, Mohanty V, Mahajan A, Oberoi A (2014) Evaluating awareness regarding oral hygiene practices and exploring gender differences among patients attending for oral prophylaxis. J Indian Soc Periodontol 18: 369-374.

11. Berlin Broner Y, levin I and Ashkenazi M (2012) Awareness of orthodontists regarding oral hygiene performance during active orthodontic treatment. Eur J Paediatr Dent 13(3): 187-191.
12. Ciancio SG (2009) The dental water jet: A product ahead of its time. Compend Contin Educ Dent 30: 7-13.

13. Krishnan V, Ambili R, Davidovitch Z, Murphy NC (2007) Gingiva and orthodontic treatment. Seminars Orthodontics 13: 257-271.

14. McCracken GI, Janssen J, Swan M, Steen N, de Jager M, et al. (2003) Effect of brushing force and time on plaque removal using a powered toothbrush. J Clin Periodontol 30: 409-413.

15. CG Jones (1997) Chlorhexidine: is it still the gold standard? Perio 2000 15: 55-62.

16. Gupta S, Saxena S (2015) Oral health attitude, knowledge and behavior of dental students of Jaipur, Rajasthan: A comparative study. J of Indian Assoc Public Health Dent 13(4): 459-464

17. Veksler AE, Kayrouz GA, Newman MG (1991) Reduction of salivary bacteria by pre-procedural rinses with chlorhexidine $0.12 \%$. J Periodontol 62: 649-651.

18. Serino G, Ström C (2009) Peri-implantitis in partially edentulous patients: association with inadequate plaque control. Clin Oral Implants Res 20(2): 169-174

19. Corbella S, Del Fabbro M, Taschieri S, De Siena F, Francetti L (2011) Clinical evaluation of an implant maintenance protocol for the prevention of peri-implant diseases in patients treated with immediately loaded full-arch rehabilitation. Int J Dent Hyg 9(3): 216222.

20. Mombelli A (2019) Maintenance therapy for teeth and implants. Perio 2000 79: 190-199.

21. Kaldahl WB, Kalkwarf KL, Patil KD, Molvar MP, Dyer JK (1996) Long term evaluation of periodontal therapy: II. Incidence of sites breaking down. J Periodontol 67: 103-108.

22. Axelsson P, Nystrom B, Lindhe J (2004) The long-term effect of a plaque control program on tooth mortality, caries and periodontal disease in adults. Results after 30 years of maintenance. J Clin Periodontol 31: 749-757.

23. Fox SC, Moriarty JD, Kusy RP (1990) The effects of scaling a titanium implant surface with metal and plastic instruments: an in vitro study. Periodontol 61(8): 491-496.

24. Yukna RA (1993) Optimizing clinical success with implants: Maintenance and care. Compend Suppl 15: S554- 561.

25. Koutsonikos A, Federico J, Yukna R (1996) Implant maintenance. J Pract Hyg 11-15. 